\title{
Références bibliographiques du dossier « L'élève, futur citoyen »
}

Sophie Condat

\section{OpenEdition}

1 Journals

Édition électronique

URL : https://journals.openedition.org/ries/173

DOI : 10.4000/ries. 173

ISSN : 2261-4265

Éditeur

France Education international

Édition imprimée

Date de publication : 1 avril 2007

Pagination : 115-126

ISBN : 978-2-85420-569-5

ISSN : 1254-4590

Référence électronique

Sophie Condat, "Références bibliographiques du dossier "L'élève, futur citoyen » », Revue internationale d'éducation de Sèvres [En ligne], 44 | avril 2007, mis en ligne le 22 juin 2011, consulté le 05 juillet 2021. URL : http://journals.openedition.org/ries/173 ; DOI : https://doi.org/10.4000/ries.173

(c) Tous droits réservés 


\section{Références bibliographiques}

\section{Sophie Condat}

Cette bibliographie, non exhaustive, concerne en majorité des travaux produits ces dix dernières années. Elle est composée de cinq parties : le cadre théorique et historique qui définit les finalités et les enjeux de l'éducation à la citoyenneté; la vision de l'UNESCO et du Conseil de l'Europe en la matière; les politiques d'éducation à la citoyenneté; les contenus : programmes et curricula, en distinguant études comparatives et études nationales; enfin, les outils destinés à l'enseignant.

Une sitographie complète la bibliographie arrêtée le 15 février 2007. Les documents mentionnés, ouvrages, articles de revues et cédéroms, sont pour la plupart consultables au centre de ressources documentaires du CIEP ou accessibles en ligne.

\section{CADRe théorique et historiQue}

\section{AUDIGIER François, L'éducation à la citoyenneté, INRP/Paris, 2000, 128 p.}

L'éducation à la citoyenneté fait partie de l'éducation scolaire. Son rôle est de tisser des relations entre les familles et les États afin d'établir un lien social et politique. Réciproquement, elle est largement influencée par les liens existant entre l'État, la société, l'institution scolaire, les familles et la religion. Comment se définit-elle? Par qui et comment doit-elle être enseignée?

BOUCHARD Nancy, LAPREE Raymond, GENDRON Claude et al., Pour un renouvellement des pratiques d'éducation morale : six approches contemporaines, Presses de l'Université du Québec/Québec, 2002, 199 p.

Cet ouvrage collectif rassemble six approches ayant pour objet l'éducation morale des jeunes du primaire et du secondaire. L'analyse de ces approches permet d'en cerner les possibilités et les limites et d'en évaluer la faisabilité.

FERRY Jean-Marc, de PROOST Séverine, de SMET François et al., L'École au défi de l'Europe : médias, éducation et citoyenneté postnationale, Université de Bruxelles/Bruxelles, 2003, 232 p. (Philosophie et société)

Avec la construction de l'Union européenne, la citoyenneté, et donc l'éducation à la citoyenneté, n'est plus strictement nationale. Elle devient aussi postnationale. Les articles rassemblés ici traitent des conditions nécessaires pour que l'école et les médias puissent jouer un rôle dans la formation d'une citoyenneté postnationale.

FRIEBEL Wim éd., Éducation à la citoyenneté européenne : approches théoriques et pratiques, Filibach Verlag/Freiburg-im-Breisgau, 1996, 261 p.

L'ouvrage s'ouvre sur un essai de clarification du concept de «citoyenneté européenne» et des termes qui lui sont généralement associés : État, nation, peuple, patrie, constitution. Dans une deuxième partie, il est fait référence à des travaux récents qui présentent deux grands types de citoyenneté coexistant actuellement en Europe, l'un se rattachant à la tradition libérale, l'autre à la tradition républicaine. La dernière partie amorce une réflexion didactique sur les enjeux et les composantes d'un projet d'éducation à la citoyenneté européenne. 
GALICHET François, L'école, lieu de citoyenneté, éditions ESF/Paris, 2005, 125 p. (Pratiques et enjeux pédagogiques)

Lorsqu'il est question d'éducation à la citoyenneté, on pense d'abord à définir ce qu'est la citoyenneté et, ensuite, à rechercher les démarches pédagogiques et didactiques pour l'enseigner. Dans cet ouvrage, François Galichet inverse cette approche en défendant une pédagogie porteuse d'un projet, d'une conception de la citoyenneté. La pédagogie peut donc devenir en elle-même et par elle-même le principe d'un modèle éthique et politique.

GALICHET François, L'éducation à la citoyenneté, Anthropos/Paris, 1998, 203 p., bibliogr.

L'ouvrage s'intéresse à l'histoire de la citoyenneté et à son enseignement. L'auteur distingue et analyse trois types de modèles et tente de dégager les conceptions politiques et pédagogiques qu'ils impliquent. En conclusion, il suggère huit mesures d'éducation à la citoyenneté.

HARPES Jean-Paul, FERRY Jean-Marc, RENAUT Alain et al., Concepts de la citoyenneté démocratique, Conseil de l'Europe/Strasbourg, 2000, 171 p., bibliogr.

Comment développer la citoyenneté démocratique en Europe? Six spécialistes livrent leur réflexion sur cette question en exposant les conditions préalables à la constitution d'une culture démocratique européenne.

LENOIR Yves, XYPAS Constantin, École et citoyenneté : un défi multiculturel, Armand Colin/Paris, 2006, 264 p., bibliogr. (Sociétales)

À l'heure où la citoyenneté européenne est une préoccupation et un enjeu majeurs, la citoyenneté nationale, confrontée aux revendications des communautés culturelles voire ethnicoreligieuses, est en crise. Quelle éducation à la citoyenneté l'école peut-elle assurer? Pour quel projet de société? Les auteurs situent l'école dans le contexte de la construction européenne, de la mondialisation et du néolibéralisme, puis évoquent le cas spécifique du modèle multiculturel québécois, celui du modèle interculturel brésilien, la lente progression de l'interculturel au Portugal et la nation face au défi de la citoyenneté européenne. En conclusion, ils livrent leur réflexion sur le rôle, la portée et les limites de l'école dans l'éducation à la citoyenneté et proposent de nouvelles pistes pédagogiques.

VERDELHAN-BOURGADE Michèle, ALVAREZ Josefina, BAUTIER Élisabeth et al., École, langage et citoyenneté, L'Harmattan/Paris, 2001, 348 p.

L'ouvrage, résultat d'un travail pluridisciplinaire rassemblant des linguistes, des juristes, des économistes et des philosophes, analyse la notion de citoyenneté dans ses rapports avec l'école. Pour les auteurs, l'éducation à la citoyenneté passe d'abord par le langage.

\section{Organisations internationales ET ÉDUCATION À LA CITOYENNETÉ}

\section{UNESCO}

Apprendre à vivre ensemble : quelle éducation pour quelle citoyenneté?, Commission nationale française pour l'UNESCO/Paris, Commission nationale marocaine pour l'éducation la science et la culture/Rabat, 2003

Ce document fait tout d'abord la synthèse du colloque international «Apprendre à vivre ensemble : quelle éducation pour quelle citoyenneté ?» organisé conjointement par les 
commissions française et marocaine pour l'UNESCO. Puis, il expose les recommandations adoptées par les participants au colloque : renforcer l'éducation aux droits de l'homme ainsi que la formation initiale et continue des enseignants; élaborer un guide des bonnes pratiques pour l'éducation à la citoyenneté; envisager des jumelages, des projets pilotes conjoints ou des programmes audiovisuels pour la promotion de la citoyenneté.

\title{
MEDEL-ANONUEVO Carolyn ed., MITCHELL Gordon ed., Citizenship, demo- cracy and lifelong learning, UNESCO Institute for Education/Hambourg, 2003, 190 p.
}

Cette publication est le résultat d'un séminaire international. Elle met en lumière des questions rarement évoquées, en s'appuyant sur les expériences de divers pays et régions du monde : l'Irlande, l'Afrique, le Belize, la Colombie, l'Afrique du Sud, les Balkans et l'Ukraine. Comment l'éducation des adultes et la formation tout au long de la vie peuvent-ils favoriser la démocratie, comment enseigner la citoyenneté? En conclusion, les auteurs soulignent l'actuel manque de connaissances, de savoirs et de compétences, indispensables à l'exercice de la démocratie tant collective qu'individuelle.

\section{Plan of Action: World Programme for Human Rights Education, UNESCO/} New York, Genève, 2006, 56 p.

En 2004, les Nations Unies ont adopté le Programme mondial en faveur de l'éducation aux droits de l'homme. La première phase du plan d'action (2005-2007) cherche à établir une vision commune de l'éducation des droits de l'homme et définit cinq facteurs de réussite comme la formation initiale et continue des enseignants ou la nécessité pour les politiques éducatives de promouvoir une approche fondée sur les droits de l'homme.

\section{TAWIL Sobhi ed., HARLEY Alexandra, Education, Conflict and Social Cohesion,} UNESCO-IBE, 2004, 433 p.

Comment l'éducation peut-elle contribuer à assurer la cohésion sociale, particulièrement dans les sociétés sortant d'une guerre civile? L'ouvrage tente de répondre à cette question en prenant comme exemples la Bosnie Herzégovine, le Guatemala, le Liban, le Mozambique, l'Irlande du Nord, le Rwanda et le Sri Lanka. Chacune de ces études analyse l'importance de la politique éducative dans la reconstruction des identités sociales et civiques et les défis auxquels sont confrontés les responsables politiques. Ces défis peuvent aller de l'élaboration des politiques linguistiques au sein de sociétés multilingues et multiculturelles à la redéfinition de la citoyenneté nationale et à la réinterprétation de l'histoire nationale.

\section{Conseil de l'Europe}

\begin{abstract}
AUDIGIER François, Éducation à la citoyenneté démocratique : concepts de base et compétences-clés pour l'éducation à la citoyenneté démocratique, Conseil de la coopération culturelle/Strasbourg, Université de Genève, 2000, 32 p. téléchargées $d u$ site $d u$ Conseil de l'Europe http://www.coe.int [consulté le 11 décembre 2006]

En 1997, le projet «Éducation à la citoyenneté démocratique» (ECD) a été lancé afin de déterminer les valeurs et les compétences nécessaires à l'individu pour qu'il devienne citoyen. Ce rapport se fait l'écho de groupes de discussion du Conseil de l'Europe sur l'éducation à la citoyenneté démocratique, groupes qui se sont réunis entre 1997 et 2000. L'auteur s'interroge sur l'intérêt croissant suscité par la citoyenneté, présente quelques débats sur ce sujet, tente de définir cette notion et décrit différentes modalités pratiques.
\end{abstract}


GOLLOB Rolf, HUDDLESTON Edward éd., KRAPF Peter, SALEMA MariaHelena et al., Éducation à la citoyenneté démocratique 2001-2004 : outil de formation des enseignants pour l'éducation à la citoyenneté démocratique et aux droits de l'Homme, Conseil de l'Europe/Strasbourg, 2005, 42 p.

Ce document insiste sur le besoin de formation des enseignants à l'éducation à la citoyenneté démocratique, les structures et les méthodes pour les former et les compétences requises pour cet enseignement.

\section{Politiques d’éducation À LA CITOYENNETÉ}

\section{Études comparatives}

ALBALA-BERTRAND Luis coord., Citoyenneté et éducation : vers une pratique significative, Perspectives (Unesco), décembre 1996, vol. XXVI, $n^{\circ}$ 4, pp. 687-829, bibliogr. La première partie du dossier présente les contextes et les perspectives de la citoyenneté dans le monde actuel et soulève certains des problèmes qui se posent quant à la pratique démocratique, au sens civique, à l'intégration nationale ou aux droits de l'homme. La seconde partie présente des tentatives concrètes et novatrices en matière d'éducation à la citoyenneté dans des régions et des cadres socio-culturels variés : la République tchèque, le Nicaragua, la République centrafricaine, l'Europe du nord.

AMADEO Jo-Ann, TORNEY-PURTA Judith, SCHWILLE John, Civic education across countries: twenty-four national case studies from the IEA civic education Project, Eburon publishers/Delft, 1999, 622 p., bibliogr.

Ces études de cas dressent un bilan synthétique de la situation de l'éducation civique dans vingt-quatre pays : contenu de l'enseignement, contexte, pratiques pédagogiques, objectifs des programmes notamment. Cette enquête a pour cadre le Projet d'éducation civique de l'International Association for the Evaluation of Educational Achievement (IEA); les données ont été collectées en 1996 et 1997 à partir d'une même grille de questions pour chaque pays.

AMADEO Jo-Ann, TORNEY-PURTA Judith, LEHMANN Rainer et al., Civic knowledge and engagement. An IEA study of upper secondary students in sixteen countries, Eburon publishers/Delft, 2002, 211 p.

L'IEA a mené une étude auprès de cinquante mille étudiants de soixante pays sur leurs connaissances en matière de droit civique et leur engagement.

BIRZEA César, KERR David, MIKKELSEN Rolf et al., Étude paneuropénne des politiques d'éducation à la citoyenneté démocratique, Conseil de l'Europel Strasbourg, 2005, 138 p.

Commencée en 2002, l'étude décrit les politiques en matière d'éducation à la citoyenneté démocratique (ECD) dans les États membres du Conseil de l'Europe. En 2003, cinq études régionales ont suivi. Le document donne aussi des recommandations et des exemples de bonne pratique pour la mise en œuvre des politiques d'ECD.

CAMILLERI GRIMA Antoinette, CANDELIER Michel, FITZPATRICK Anthony et al., La diversité linguistique en faveur de la citoyenneté démocratique en Europe : vers un document-cadre pour des politiques linguistiques éducatives, Conseil de l'Europe/Strasbourg, 2001, 185 p. 
L'ouvrage est fondé sur les conclusions d'une conférence portant sur le rapprochement des peuples d'Europe à travers l'apprentissage des langues. Rapports, discours et discussions défendent, pour la plupart, l'idée que la diversité linguistique est en mesure de jouer un rôle éminent dans la promotion de la citoyenneté démocratique.

\section{Conseil de l'Europe, Textes adoptés sur l'éducation à la citoyenneté démocratique et aux droits de l'homme, La Documentation française/Paris, 2003, 81 p.}

La Recommandation aux États membres relative à l'éducation à la citoyenneté démocratique a été adoptée par le Comité des ministres en octobre 2002. Le document présent est le principal résultat des travaux menés par le Conseil de l'Europe depuis 1997 sur cette question. Il présente le programme de la mise en œuvre des politiques d'éducation à la citoyenneté dans les États membres.

\section{COGAN John J., DERRICOTT Ray, Citizenship for the 21st century: an inter-} national perspective on education, Kogan Page/Londres, 2000, 256 p., index

L'ouvrage cherche à démontrer l'importance d'une éducation à la citoyenneté en s'appuyant sur des exemples d'Asie, d'Europe et d'Amérique du Nord. Il propose également quelques suggestions pour développer au mieux cette politique.

\section{Eurydice: Réseau européen d'information sur l'éducation, L'éducation à la citoyenneté à l'école en Europe, Eurydice/Bruxelles, 2005, 88 p.}

Cette étude analyse les modalités d'éducation à la citoyenneté mises en place aux niveaux primaire et secondaire dans trente pays européens. Après un panorama des différentes définitions de la citoyenneté, cette analyse comparative s'intéresse aux objectifs, aux contenus et aux approches de l'éducation à la citoyenneté, à la participation des élèves à la vie de l'école et de la communauté locale et aux modalités d'évaluation. Les deux derniers chapitres portent sur la formation des enseignants dans ce domaine et sur la dimension européenne de l'éducation à la citoyenneté.

HAHN Carole, KERR David, KENNEDY Kerry J. et al., "Political and citizenship education ", Oxford studies in comparative education, 2004, vol. 14, $n^{\circ} 2,144$ p., bibliogr.

Basé sur les études menées par l'IEA, ce numéro analyse les concepts et les réalités de l'éducation à la citoyenneté à partir de recherches internationales portant sur les États-Unis, le RoyaumeUni, l'Australie, Hong-Kong, la République tchèque et l'Allemagne.

\section{KENNEDY Kerry J., Citizenship Education and the Modern State, Routledgefalmer/ London, 1997, 196 p.}

Avec la transformation rapide de la nation à la fin du XXe siècle, les États ont focalisé leur attention sur le curriculum comme moyen de maintenir la cohésion au sein de la société. Cet ouvrage veut donner aux éducateurs les clés d'information pour les aider à comprendre les initiatives actuelles prises par certains pays en matière d'éducation à la citoyenneté et leur mise en œuvre.

O'SHEA Karen, Éducation à la citoyenneté démocratique : les politiques et les instruments législatifs. Séminaire international. Strasbourg 6 et 7 décembre 2001, La Documentation française/Paris, 2003, 62 p.

Ce rapport d'un séminaire portant sur le développement et la promotion au niveau européen d'une politique de l'éducation à la citoyenneté démocratique s'articule autour de trois objectifs : faire le point sur les politiques et les pratiques concernant l'éducation à la citoyenneté démocratique (ECD), promouvoir la coopération européenne entre pays, organisations et praticiens, et formuler des propositions sur la mise en place de l'ECD. 
TORNEY-PURTA Judith, LEHMANN Rainer, OSWALD Hans, SCHULZ Wolfram, Citizenship and education in twenty-eight countries. Civic knowledge and engagement at age fourteen, Eburon publishers/Delft, 2001, 237 p., bibliogr.

L'ouvrage rend compte des résultats de la seconde phase de l'étude sur l'éducation civique menée par l'IEA. En 1999, des chercheurs ont interrogé, dans vingt-huit pays, quatre-vingt dix mille élèves de quatorze ans, ainsi que des enseignants et des chefs d'établissement sur la démocratie, l'identité nationale, la diversité et la cohésion sociale. L'ouvrage dresse un tableau comparatif des connaissances des élèves, de leurs compétences et de leurs comportements liés à la citoyenneté. Il donne aussi un aperçu des activités civiques dans lesquelles ils s'engagent.

\title{
Études nationales
}

LEHOUX Jean-François coord., JULIEN Mélanie, IBRAHIM Amir et al., Rapport annuel sur l'état et les besoins de l'éducation 2005-2006 : agir pour renforcer la démocratie scolaire, Conseil supérieur de l'éducation/Québec, 2006, 111 p., bibliogr., annexes, tableaux, graphiques

Dans son rapport annuel, le Conseil supérieur du Québec affirme la nécessité de renforcer les pratiques démocratiques au sein du système scolaire québécois. Il propose cinq orientations : éduquer à la citoyenneté pour contribuer à la pérennité de la démocratie; recentrer la démocratie scolaire sur les élèves; s'assurer d'une compréhension commune du partage des pouvoirs et des responsabilités en éducation; promouvoir la richesse de la contribution des parents dans toute leur diversité; renforcer la légitimité du gouvernement scolaire.

LACROIX Jean-Michel dir., LINTEAU Paul-André, Vers la construction d'une citoyenneté canadienne, Presses de la Sorbonne nouvelle/Paris, 2006, 242 p.

L'ouvrage analyse les déclinaisons religieuses et linguistiques du multiculturalisme canadien dans le champ de l'éducation. Il donne des éléments de réflexion sur une société civile soucieuse de bâtir une citoyenneté interculturelle et laïque au service de l'intérêt national.

MUHIMPUNDU Félicité, Éducation et citoyenneté au Rwanda, L'Harmattan/ Paris, 2002, $268 p$.

L'histoire du Rwanda révèle une non-intégration des notions fondamentales de la citoyenneté. Pour l'auteur, le système éducatif peut créer un sentiment de citoyenneté et contribuer à la paix.

\section{CONTENUS : PRATIQUeS, PROGRAMMES ET CURRICULA}

\section{Études comparatives}

\begin{abstract}
ALEXANDER Robin, HOFMANN Jan, PRAIRAT Eirick, L'apprentissage de la civilité à l'école : regards croisés, ministère de la Jeunesse, de l'Éducation et de la Recherche. DESCO : Direction de l'enseignement scolaire, 2003, 36 p.

Dans cette publication de la DESCO, des enseignants français, anglais et allemands portent un regard croisé sur l'apprentissage de la civilité à l'école dans leurs pays respectifs. Trois interventions ont suivi l'ouverture de la conférence-débat. Celle de l'enseignant anglais a porté sur l'apprentissage, la civilité et la culture dans une perspective internationale; la contribution allemande, sur les valeurs dans la tradition de l'enseignement allemand; la française, sur les valeurs à l'heure de la désacralisation de l'ordre scolaire.
\end{abstract}


AUDIGIER François, Pour une approche comparée de l'éducation à la citoyenneté dans quelques curriculums européens, 34 pages téléchargées du site http://www. proformar.org [consulté le 06/02/2007]

Lors du séminaire de Lille en septembre 2003 «Éduquer à la citoyenneté active en Europe», François Audigier a tenu une conférence sur l'approche comparée de l'éducation à la citoyenneté dans quelques curricula européens. Le document présente tout d'abord quelques préalables sur les systèmes éducatifs et la citoyenneté et rappelle brièvement les spécificités de l'éducation à la citoyenneté.

BIRZEA César, CECCHINI Michela, HARRISON Cameron et al., Tool for quality assurance of education for democratic citizenship in schools, Conseil de l'Europel Strasbourg, 2005, 124 pages téléchargées du site http://www.coe.int [consulté le 11 décembre 2006]

Conçue comme un document de référence, cette publication a été réalisée pour combler l'écart entre les politiques d'éducation à la citoyenneté démocratique et leur mise en application au sein de nombreux pays. Plusieurs points sont abordés, comme le rôle de l'éducation à la citoyenneté à l'école ou sa planification scolaire.

Consortium of Institutions for Development and Research Education (CIDREE). The Standing International Conference of Central and General Inspectorates of Education (SICI), Raising the effectiveness of citizenship education: the perspectives of curriculum developers, school inspectors and researchers, CIDREE-SCI/Brussels, 2002, 52 pages téléchargées du site http://www.sici.org.uk [consultéle 11 décembre 2006] Cet atelier organisé par le CIDREE a repris les réflexions de l'atelier sur l'évaluation de l'éducation à la citoyenneté, organisé par la SICI en 1999, en privilégiant trois angles de vue : ceux des concepteurs de curricula, des inspecteurs de l'éducation et des chercheurs. Il a conclu à la nécessité de créer un site web interactif sur l'éducation à la citoyenneté.

KERR David, IRELAND Eleanor, LOPES Joana et al., Making citizenship education real. Citizenship education longitudinal study: second annual report. First longitudinal survey, Dfes/London, 2004, 154 pages téléchargées du site http://www.dfes.gov.uk [consulté le 11 décembre 2006]

Le rapport souligne les approches émergentes d'éducation à la citoyenneté dans les établissements de Grande-Bretagne durant l'année 2002-2003. Il identifie et analyse les éléments qui influencent sa mise en place et souligne ses effets pour les écoles, les enseignants et les décideurs politiques.

MEDIONI Maria-Alice, Construire ses savoirs. Construire sa citoyenneté. De l'école à la cité, Chronique sociale/Lyon, 1996, 315 p. (Pédagogie formation. L'essentiel) Comment penser le savoir et la citoyenneté dans les banlieues et nos sociétés? Comment mettre en œuvre la dialectique construction du savoir/exercice de la citoyenneté? Pour répondre à ces interrogations qui débordent largement le cadre des banlieues et le champ de l'école, l'ouvrage propose des pistes de réflexion, des projets innovants et des stratégies concrètes.

\footnotetext{
RAVEAUD Maroussia, De l'enfant au citoyen : la construction de la citoyenneté à l'école en France et en Angleterre, PUF/Paris, 2006, 209 p.

Quelle est la contribution de l'école dans la formation des citoyens? Cette étude comparative sur des écoles primaires françaises et anglaises met en lumière les spécificités politiques, philosophiques et scolaires de ces deux pays. Les dynamiques à l'œuvre dans les classes sont révélatrices des valeurs nationales. Il apparaît que la citoyenneté n'est pas un invariant universel, mais une construction sociale spécifique à une culture donnée.
} 
RAVEAUD Maroussia, "Minorités-ethnicité et citoyenneté : les modèles français et anglais sur les bancs de l'école", Revue française de pédagogie, septembre 2003, $n^{\circ}$ 144, pp. 19-28, bibliogr.

La scolarisation des enfants issus de l'immigration renvoie aux modèles nationaux d'intégration et de construction identitaire, des problématiques qui dépassent le cadre scolaire. L'article compare la situation en France et en Angleterre, deux pays aux modèles d'intégration très distincts : le modèle républicain et le modèle multiculturel.

\section{SCOTT David, LAWSON Helen, Citizenship Education and the Curriculum} (International Perspectives on Curriculum Studies), Hardcover/Canada, 2002, 200 p.

Les auteurs abordent plusieurs questions, parmi lesquelles la relation entre l'éducation à la citoyenneté et le curriculum, les différents modèles d'éducation à la citoyenneté et l'évaluation. L'ouvrage propose une approche comparative de l'éducation à la citoyenneté en prenant comme exemples la Nouvelle-Zélande, l'Angleterre, l'Islande, Israël et l'Irlande.

The Dutch Ministry of Education, Culture and Science, Citizenship - made in Europe: living together starts at school, Zoetermeer: The Dutch Ministry of Education, Culture and Science, 2004, 130 pages téléchargées du site http://www. hrea.org [consulté le 11 décembre 2006]

S'il y a un large consensus entre les membres de l'Union européenne sur l'urgence d'une éducation à la citoyenneté pour assurer la cohésion sociale, l'étude révèle que les décideurs politiques n'ont pas toujours connaissance des politiques actuellement en œuvre dans ce domaine mais aussi qu'il existe souvent un fossé entre les intentions politiques et leur mise en pratique. La dernière partie de l'étude donne les conclusions d'une réunion d'experts, selon lesquelles les enfants devraient commencer à apprendre à vivre ensemble le plus tôt possible afin d'acquérir des connaissances, des compétences et des comportements.

TOMASEVSKI Katarina, Manual on rights-based education: global human rights requirements made simple: collaborative project between the UN Special Rapporteur on the right to education and UNESCO Asia and Pacific Regional Bureau for Education, UNESCO/Paris, 2004, 60 pages téléchargées du site http://www.unescobkk.org [consulté le 11 décembre 2006]

Conçu comme un outil de référence pour les décideurs politiques, les praticiens de l'éducation et pour ceux qui travaillent dans le domaine de la coopération internationale, ce manuel définit, au plan national, des grandes orientations en matière d'éducation aux droits de l'homme.

\section{Études nationales}

Advisory Group on Citizenship, Education for citizenship and the teaching of democracy in schools, QCA/London, 1998, 88 pages téléchargées du site http:// www.dfes.gov.uk [consulté le 11 décembre 2006]

La commission consultative est à l'origine de l'introduction de l'éducation à la citoyenneté dans les programmes d'enseignement anglais.

CHARBONNIER Daniel, "L'école anglaise face au défi de la citoyenneté », La Revue de l'Inspection générale, février 2004, $n^{\circ}$ 1, pp. 66-74, bibliogr.

À l'heure où la société anglaise est en train de devenir l'une des sociétés les plus multiculturelles et multiconfessionnelles d'Europe, le système éducatif s'interroge sur l'articulation entre l'éducation à la citoyenneté et l'enseignement religieux, deux matières obligatoires inscrites dans le cursus scolaire anglais. 
CONDETTE-CASTELAIN S., La participation des lycéens à la vie de leur établissement, ANRT, Lille, 2005

\section{Croatian commission for UNESCO, Peace and human rights for croatian primary} schools, Kikagraf/Zagreb, 2000, 54 p.

Ce livret présente un projet visant à promouvoir l'éducation à la paix et aux droits de l'homme dans les écoles primaires croates par l'élaboration d'un programme et de matériels éducatifs.

FLONNEAU Monique, L'éducation à la citoyenneté, Nathan/Paris, 2004, 94 p., (Les pratiques de l'éducation)

L'ouvrage présente les fondements d'une démarche pédagogique centrée sur la prise de conscience de l'enfant, à partir de situations du quotidien. Il propose une analyse théorique du sujet (le civisme et la citoyenneté, la neutralité de l'enseignant, l'enfant citoyen) ainsi que des démarches pédagogiques pour l'enseignant.

KARAKATSANI Despina, «La citoyenneté dans le système éducatif grec : matières, activités et pratiques", Spirale : revue de recherches en éducation, 2004, $n^{\circ} 34$, pp. $157-170$

L'article analyse les nouveaux besoins socio-politiques et tendances de l'enseignement civique en Grèce.

LAMARRE Jean-Marc, "L'éducation civique et morale à l'école est-elle encore possible?», Recherche et formation, 2006, $n^{\circ}$ 52, pp. 29-41

À une époque où l'individualisme règne, l'auteur s'interroge sur les moyens d'assurer aux enfants une éducation civique et morale. Celle-ci est possible grâce aux pratiques scolaires du récit et du débat à l'école primaire.

LEVINSON Bradley A.U., "Hopes and challenges for the new civic education in Mexico: toward a democratic citizen without adjectives", International journal of educational development, mai 2004, vol. 24, $n^{\circ} 3$, pp. 269-282, bibliogr.

L'article présente les principaux objectifs et thèmes d'une ambitieuse réforme du programme d'éducation civique à Mexico. Il souligne également l'importance de quelques-uns des défis nationaux auxquels les politiques sont confrontés.

\section{PERRENOUD Philippe, L'École est-elle encore le creuset de la démocratie?,} Chronique sociale/Lyon, 2003, 192 p. (Pédagogie - Formation)

Face à la montée de la violence, de l'individualisme et de l'affaiblissement du lien social, l'école est-elle encore le creuset de la démocratie? Peut-elle raisonnablement jouer ce rôle? L'auteur considère que seule une véritable éducation à la citoyenneté permettra de relever le défi, ce qui nécessite de donner de vrais moyens à l'école, de réformer les programmes d'enseignement et de former les enseignants. Éduquer à la citoyenneté, c'est faire acquérir aux élèves les valeurs fondamentales (égalité, solidarité, justice, diversité, liberté), les initier à la culture du débat et au respect de la diversité des opinions, leur inculquer un minimum de pratique de la démocratie et de la responsabilité, leur apprendre à vivre ensemble.

WILDE Stéphanie, Citizenship Education in Germany: not doing it by the book, Symposium Books/Oxford, 2004, 142 p., bibliogr. (Monographs in International Education)

Cette étude traite de l'éducation à la citoyenneté, de l'éducation politique et de l'éducation interculturelle en Allemagne. Elle s'appuie notamment sur des études de cas réalisées en 2000-2002 auprès d'établissements secondaires des Länder de l'Ouest comme de l'Est. 


\section{France}

Ministère de l'éducation nationale, de l'enseignement et de la recherche, Les textes de références sur l'éducation à la citoyenneté, http://eduscol.education.fr/D0004/ vtcref01.htm? rub $=4$

Sur EduSCOL, le site pédagogique du ministère de l'éducation nationale, de l'enseignement et de la recherche, un ensemble de textes réglementaires et législatifs sur l'éducation à la citoyenneté.

Ministère de l'éducation nationale, de l'enseignement supérieur et de la recherche, B.O., 25 novembre 2004, $n^{\circ} 43$, Éducation à la citoyenneté : enseignement élémentaire et secondaire, http://www.education.gouv.fr/bo/2004/43/MENE0402540N.htm Le texte officiel adressé aux recteurs, inspecteurs et directeurs des services départementaux de l'Éducation nationale concernant l'éducation à la citoyenneté dans l'enseignement élémentaire et secondaire.

\section{OUTILS POUR L'ENSEIGNANT}

AUDIGIER François, GILLMAN Jean, MITROFAN Rodica et al., Stratégies pour une éducation civique au niveau de l'enseignement primaire et secondaire, Conseil de l'Europe/Strasbourg, 2000, 156 p.

Ce guide méthodologique, réalisé dans le cadre du projet du Conseil de la coopération culturelle «Démocratie, droits de l'homme, minorités : les aspects éducatifs et culturels», s'adresse aux enseignants du primaire et du secondaire. Il définit tout d'abord les notions et principes de base, puis, analyse les problèmes sociaux et les aspects politiques et juridiques que pose l'éducation civique.

AUDIGIER François, LAGELEE Guy dir., Éducation civique et initiation juridique dans les collèges, INRP/Paris, 1996, 267 p., bibliogr. (Didactique des disciplines) L'ouvrage vise à initier les élèves à quelques concepts juridiques en proposant aux enseignants des informations, des réflexions mais aussi des séquences et des activités pédagogiques.

AVGERI Parthenia, GUILLOBEZ Thomas, JAGODNIK Edouard et al., Citoyenneté démocratique et droits de l'homme : culture et citoyenneté européennes, Foucher/Paris, 2006, 94 p. (Plein Pot Horizons européens)

Dans l'intérêt de la cohésion sociale en Europe et d'une identité européenne commune, l'école doit informer les élèves des droits et des responsabilités qu'implique la citoyenneté européenne. Destiné aux lycéens, cet ouvrage se veut un outil pédagogique transversal, regroupant et développant des connaissances fondamentales.

DUTHEL Dominique, PUEL Gilles, MATHE Bernard et al., Des ressources pour de nouvelles pratiques au lycée en sciences économiques et sociales, histoiregéographie, éducation civique juridique et sociale, CRDP Midi-Pyrénées/MidiPyrénées, 2000, 126 p. (Citoyenneté - Europe - TICE)

Les thèmes de la citoyenneté et de l'Europe sont présents dans les programmes de plusieurs disciplines : les sciences économiques et sociales, l'histoire-géographie, l'éducation civique, juridique et sociale. Ce volume propose ressources, idées et supports pour exploiter pédagogiquement ces notions.

LE GAL Jean, Les droits de l'enfant à l'école : pour une éducation à la citoyenneté, De Boeck/Bruxelles, Belin/Paris, 2002, 214 p., bibliogr. (Comprendre)

L'ouvrage donne aux équipes pédagogiques des éléments pour les aider à mettre en place une éducation à la citoyenneté grâce à une participation active et responsable des enfants (conseils 
de classe et d'école, organisation des libertés et des responsabilités, démarche participative et projets). L'ouvrage aborde la citoyenneté démocratique, la citoyenneté de l'enfant, les droits de l'enfant et plaide en faveur d'une citoyenneté participative à l'école.

L'éducation civique, une dominante transversale : cycles 1, 2 et 3, CRDP Créteil/Créteil, 2003, 416 p. (Professeur aujourd'hui)

Les auteurs, professeurs de l'IUFM de Créteil, montrent que l'éducation civique touche toutes les disciplines (l'histoire, la géographie, les sciences, le théâtre, l'éducation physique et sportive), proposent une analyse critique des textes de références et, enfin, présentent des pratiques et des projets pédagogiques.

LELEUX Claudine, Éducation à la citoyenneté. Tome 1 : les valeurs et les normes de 5 à 14 ans, De Boeck/Bruxelles, 2006, 207 p. (Outils pour enseigner)

L'éducation morale et l'éducation à la citoyenneté sont formulées en termes de compétences transdisciplinaires et interdépendantes, tant cognitives que participatives. La démarche est illustrée par une trentaine de situations pédagogiques conçues pour l'école primaire et le début du cycle secondaire.

MARZOUK Abdellah, KABANO John, COTE Pauline, Éduquer à la citoyenneté à l'école : guide pédagogique, Éditions logiques/Montréal, 2000, 123 p. (Théories et pratiques dans l'enseignement)

Tout en s'attachant aux thèmes fondamentaux de l'éducation à la citoyenneté, ce guide propose aux enseignants un modèle pédagogique à suivre ainsi que des thèmes et des activités. Une partie théorique aborde le concept de citoyenneté, l'école et la formation du futur citoyen, l'apprentissage de la citoyenneté. Le dernier chapitre porte sur l'application pédagogique.

Ministère de l'Éducation nationale de la recherche et de la technologie, L'éducation à la citoyenneté, INRP/Paris, 1998, 198 p., bibliogr. (Pratiques innovantes)

L'ouvrage présente la réflexion et les actions mises en œuvre dans les classes et les établissements en matière d'éducation à la citoyenneté. L'objectif est non seulement de reconnaître la créativité des équipes d'enseignants mais aussi de donner à d'autres équipes l'envie d'innover et des outils pour le faire.

\section{REARDON Betty A., L'éducation pour une culture de la paix : la prise en compte du genre, UNESCO/Paris, 2003, 178 p.}

Pour l'UNESCO, l'éducation doit contribuer à une culture de la paix. L'auteur estime que les programmes d'enseignement doivent promouvoir des valeurs et des comportements allant dans le sens d'une culture de la paix tels que le dialogue, la recherche de consensus et la non-violence. Cet ouvrage, destiné aux enseignants du secondaire, se veut un outil pédagogique de formation. La première partie s'intéresse aux concepts et aux finalités de l'éducation pour une culture de la paix; la seconde, à ses dimensions professionnelles et pédagogiques. Des références bibliographiques et une liste de sites Internet permettent d'approfondir le sujet.

ROCHE Anne, BASSET Yves, FAYOL Michel et al., L'éducation civique aujourd'hui : dictionnaire encyclopédique, ESF/Paris, 2002, 455 p. (Pédagogies/ outils)

Ce dictionnaire, fruit d'un travail collectif, est destiné avant tout aux lycéens, ainsi qu'aux maitres en formation. Pour chaque terme ou notion choisis dans les quatre domaines traités (éducation civique, morale, juridique et politique), les auteurs présentent l'origine et le sens de la notion avant de dégager la problématique et de présenter des éléments de réponse. 
UNESCO : Organisation des Nations Unies pour l'éducation, la science et la culture, La défense au service de la paix, CNDP/Paris, UNESCO/Paris, 2005

Rédigé sous forme de fiches par des spécialistes de la question, ce manuel d'éducation à la citoyenneté s'adresse aux professeurs du secondaire. Il traite de la relation de la défense et de la paix, permet de comprendre et de réfléchir aux fondements de la paix et de la sécurité internationale. Un glossaire juridique, une bibliographie et une cartographie complètent le manuel.

UNESCO : Organisation des Nations Unies pour l'éducation, la science et la culture, Pour une éducation à la citoyenneté=Education for Citizenship=Educacion para la ciudadania, UNESCO/Paris, 2001, cédérom

Accessible en trois langues (anglais, français et espagnol), ce cédérom conçu et produit par l'UNESCO est destiné à aider les enseignants à promouvoir les valeurs de citoyenneté dans les écoles. Il propose un glossaire de 180 termes, des textes d'orientation, des guides méthodologiques, des activités d'apprentissage ainsi qu'une sélection de références bibliographiques et de vidéos produites ou coproduites par des organisations internationales.

VINCENT Jean-François, Éduquer à la citoyenneté, Delagrave/Paris, 2006, 107 p. (Guides de poche pour l'enseignant)

Pour l'auteur, éduquer à la citoyenneté n'est pas seulement transmettre des connaissances mais aussi donner à l'élève les moyens d'exercer une citoyenneté active. L'ouvrage se divise en six objectifs : construire l'estime de soi, s'approprier des valeurs nécessaires pour vivre ensemble, faire l'apprentissage de la démocratie participative, construire le rapport à la loi, articuler droits et obligations et enfin éduquer aux médias.

\section{SITOGRAPHIE}

\section{UNESCO, L'éducation à la citoyenneté}

Ce site recense les activités, les publications, les ressources en ligne et les documents de base sur ce que fait l'UNESCO en matière d'éducation à la citoyenneté. http://portal.unesco.org/education/fr

\section{Conseil de l'Europe, La Division de l'éducation à la citoyenneté}

Un site sur l'éducation à la citoyenneté démocratique : les définitions et les enjeux, la politique du Conseil de l'Europe, sa mise en place dans les États membres. En ligne également, des publications et des documents pédagogiques, une sélection de liens Internet et une rubrique actualités. http://www.coe.int/t/dg4/education/edc/Interinstitutional_Cooperation/Default_Fr.asp

\section{Conseil de l'Europe, Éducation pour la citoyenneté démocratique}

En ligne sur le site du Conseil de l'Europe, une bibliographie recense des documents $\mathrm{du}$ Conseil de l'Europe ainsi qu'une sélection d'ouvrages et d'articles sur le sujet. http://www.coe. int/te/cultural_co-operation

Eurydice : Réseau européen d'information sur l'éducation, L'éducation à la citoyenneté en Europe : descriptions nationales

Cette publication donne une information détaillée sur l'éducation à la citoyenneté démocratique dans l'enseignement primaire et secondaire de vingt-neuf pays européens. Les fiches descriptives nationales sont toutes structurées ainsi : citoyenneté et politiques éducatives; ECD dans les programmes d'études; culture de l'école et participation dans la communauté locale; évaluation; compétences des enseignants et soutien; dimension européenne/internationale de l'éducation à la citoyenneté; réformes et débats en cours; références. http://www.eurydice.org 